\title{
Asymptotic behavior of discrete semigroups of bounded linear operators over Banach spaces
}

Shuhong Tang ${ }^{\mathrm{a}}$, Akbar Zada ${ }^{\mathrm{b}}$, Habiba Khalid ${ }^{\mathrm{b}}$, Tongxing $\mathrm{Li}^{\mathrm{c}, \mathrm{d}, *}$

${ }^{a}$ School of Information and Control Engineering, Weifang University, Weifang, Shandong 261061, P. R. China.

${ }^{b}$ Department of Mathematics, University of Peshawar, Peshawar 25000, Pakistan.

${ }^{c}$ LinDa Institute of Shandong Provincial Key Laboratory of Network Based Intelligent Computing, Linyi University, Linyi, Shandong 276005, P. R. China.

${ }^{d}$ School of Informatics, Linyi University, Linyi, Shandong 276005, P. R. China.

\begin{abstract}
Assume that $\vartheta_{j}$ is the solution of the nonhomogeneous Cauchy problem

$$
\vartheta_{j+1}=\rho(1) \vartheta_{j}+f(j+1), \quad \vartheta_{0}=0,
$$

where $\rho(1)$ is the algebraic generator of the discrete semigroup $\mathbf{T}=\left\{\rho(j): j \in \mathbb{Z}_{+}\right\}$acting on a complex Banach space $\Delta$. Suppose further that $\mathbf{A A P}_{0}^{\mathrm{r}}\left(\mathbb{Z}_{+}, \Delta\right)$ is the space of asymptotically almost periodic sequences with relatively compact ranges. We prove that the system

$$
u_{j+1}=\rho(1) u_{j}
$$

is uniformly exponentially stable if and only if for each $f \in \mathbf{A A P}_{0}^{\mathrm{r}}\left(\mathbb{Z}_{+}, \Delta\right)$ the solution $\vartheta_{j} \in \mathbf{A A P}_{0}^{\mathrm{r}}\left(\mathbb{Z}_{+}, \Delta\right)$. (C)2017 All rights reserved.
\end{abstract}

Keywords: Banach space, difference equation, uniform exponential stability, almost periodic sequence, relatively compact space.

2010 MSC: 39A10, 39A30.

\section{Introduction}

Assume that $A$ is a bounded linear operator over a complex Banach space $\Delta$. A well-known result of Daleckiǐ and Kreǐn [9] and Krěn [12] shows that the system $\dot{\vartheta}(t)=A \vartheta(t)$ is uniformly exponentially stable if and only if for each $\alpha \in \mathbb{R}$ and each $b \in \Delta$ the solution of the Cauchy problem

$$
\left\{\begin{array}{l}
\dot{\eta}(t)=A \eta(t)+e^{i \alpha t} b, \\
\eta(0)=0
\end{array}\right.
$$

\footnotetext{
*Corresponding author

Email addresses: wfxytang@163.com (Shuhong Tang), zadababo@yahoo.com (Akbar Zada), habibakhalid1994@gmail.com (Habiba Khalid), litongx2007@163.com (Tongxing Li)

doi:10.22436/jmcs.017.02.12
} 
is bounded. One can find the proof of this classic result in [2]. This result can also be extended for strongly continuous bounded semigroups, we refer the reader to $[5,14,16]$. Under a slightly different assumption, the result on stability was also preserved for any strongly continuous semigroups acting on complex Hilbert spaces; see, for instance, $[13,15]$ and the references cited therein. For counter-examples, see $[4,10]$. In $[7,18]$, the same results were extended for square size matrices in both continuous case and discrete case.

To the best of our knowledge, so far, there are few related results regarding the study of discrete systems, discrete semigroups, and discrete evolution families. Very recently, Buşe et al. [6] considered the uniform exponential stability of discrete nonautonomous systems on the space of sequences denoted by $\mathcal{C}_{00}\left(\mathbb{Z}_{+}, \Delta\right)$, where $\mathbb{Z}_{+}$is the set containing all nonnegative integers. Ahmad et al. [1], Khan et al. [11], and Zada et al. [19] studied uniform exponential stability of discrete semigroups, whereas Wang et al. [17] gave a result on the uniform exponential stability of discrete evolution families on space of p-periodic sequences denoted by $\mathcal{P}_{0}^{\mathfrak{p}}\left(\mathbb{Z}_{+}, \Delta\right)$. In particular, Zada et al. [19] proved that the system $\vartheta_{j+1}=\rho(1) \vartheta_{j}$ is uniformly exponentially stable if and only if for each q-periodic bounded sequence $\varphi(j)$ with $\varphi(0)=0$ and each $\alpha \in \mathbb{R}$ the solution of the Cauchy problem

$$
\left\{\begin{array}{l}
\eta_{j+1}=\rho(1) \eta_{j}+e^{i \alpha(j+1)} \varphi(j+1), \\
\eta_{0}=0,
\end{array}\right.
$$

is bounded, where the meaning of $\rho(1)$ will be explained in the next section.

The principal objective of this article is to extend the results of [19] to the space of asymptotically almost periodic sequences with relatively compact ranges denoted by $\mathbf{A A P}_{0}^{\mathrm{r}}\left(\mathbb{Z}_{+}, \Delta\right)$. For the periodic and almost periodic sequences, we refer the reader to $[3,8]$ and the references cited there.

\section{Notation and preliminaries}

Suppose that $\mathcal{L}(\Delta)$ is the Banach algebra of all bounded linear operators on $\Delta$. The norms on $\Delta$ and $\mathcal{L}(\Delta)$ will be denoted by $\|\cdot\|$. We recall that $\mathbf{T}=\left\{\rho(j): j \in \mathbb{Z}_{+}\right\}$of bounded linear operators acting on $\Delta$ is a discrete semigroup if it satisfies the following properties:

(1) $\rho(0)=I$, where I is the identity operator on $\Delta$;

(2) $\rho(j+m)=\rho(j) \rho(m)$ for all $j, m \in \mathbb{Z}_{+}$.

Let $\rho(1)$ be the algebraic generator of the semigroup $T$. It is clear that $\rho(\mathfrak{j})=\rho^{j}(1)$ for all $j \in \mathbb{Z}_{+}$. The growth bound of $\mathbf{T}$ is denoted by

$$
\omega_{0}(\mathbf{T})=\inf \left\{\omega \in \mathbb{R}:\|\rho(j)\| \leqslant M_{\omega} e^{\omega j} \text { for all } j \in \mathbb{Z}_{+} \text {and some } M_{\omega}>0\right\} .
$$

The family $\mathbf{T}$ is uniformly exponentially stable if $\omega_{0}(\mathbf{T})$ is negative or equivalently, if there exist two positive constants $M$ and $\omega_{1}$ such that

$$
\|\rho(j)\| \leqslant M e^{-\omega_{1} j}, \quad \forall j \in \mathbb{Z}_{+} .
$$

Let $\operatorname{BUC}(\mathbb{Z}, \Delta)$ be the Banach space of all $\Delta$-valued, bounded, and uniformly convergent sequences on $\mathbb{Z}$, endowed with the sup-norm. Now, we consider several subspaces of $\mathbf{B U C}(\mathbb{Z}, \Delta)$. Let $\mathbf{C}_{0}(\mathbb{Z}, \Delta)$ be the space consisting of all $\Delta$-valued sequences vanishing at infinity, $\mathbf{P}_{\mathrm{q}}(\mathbb{Z}, \Delta)$ with $\mathrm{q} \geqslant 2$ be the space consisting of all q-periodic sequences, and $\mathbf{A P}(\mathbb{Z}, \Delta)$ be the smallest closed subspace of $\mathbf{B U C}(\mathbb{Z}, \Delta)$ which contains all sequences of the form $j \rightarrow e^{i \mu j} v: \mathbb{Z} \rightarrow \Delta, \mu \in \mathbb{R}, v \in \Delta$. $\operatorname{AAP}^{r}(\mathbb{Z}, \Delta)$ is the space consisting of all sequences $x(\mathfrak{j})$ with relatively compact ranges for which there exist a sequence $y(\mathfrak{j}) \in \mathbf{A P}(\mathbb{Z}, \Delta)$ and a sequence $z(\mathfrak{j}) \in \mathbf{C}_{0}(\mathbb{Z}, \Delta)$ such that $x(\mathfrak{j})=y(\mathfrak{j})+z(\mathfrak{j})$.

If $\mathbf{F}(\mathbb{Z}, \Delta)$ is a suitable Banach space, then $\mathbf{F}(\mathbb{Z}, \Delta)$ is invariant under the operator $\mathcal{S}(\cdot)$ defined by

$$
(\mathcal{S} * g)(r)=\rho(j) g(r-j), \quad r \in \mathbb{Z}_{+},
$$

and $\mathbf{S}=\{\mathcal{S}(\mathfrak{j})\}_{\mathbf{j} \in \mathbb{Z}_{+}}$is the evolution semigroup on the space $\mathbf{F}(\mathbb{Z}, \Delta)$. If $\mathbf{T}$ is q-periodic and $\mathbf{F}(\mathbb{Z}, \Delta)=$ 
$\mathbf{P}_{\mathbf{q}}(\mathbb{Z}, \Delta)$ (or $\mathbf{A P}(\mathbb{Z}, \Delta)$ ), then $\mathbf{S}=\{\mathcal{S}(\mathfrak{j})\}_{\mathbf{j} \in \mathbb{Z}}$ acts on $\mathbf{P}_{\mathbf{q}}(\mathbb{Z}, \Delta)$ (or $\mathbf{A P}(\mathbb{Z}, \Delta)$ ). Furthermore, if $\mathbf{T}$ is asymptotically almost periodic with relatively compact ranges and for each $v \in \Delta, \lim _{j \rightarrow 0} \rho(\mathfrak{j}) v=v$, uniformly for $j \in \mathbb{Z}_{+}$, then $\mathbf{S}$ is defined on $\mathbf{A A P}^{r}(\mathbb{Z}, \Delta)$.

\section{Exponential stability and action of convolution on $\mathbf{P}_{\mathbf{q}}(\mathbb{Z}, \Delta) \oplus \mathrm{C}_{0}(\mathbb{Z}, \Delta)$}

Let $\mathbf{F}_{\mathbf{q}}(\mathbb{Z}, \Delta)=\mathbf{P}_{\mathbf{q}}(\mathbb{Z}, \Delta) \oplus \mathbf{C}_{0}(\mathbb{Z}, \Delta)$. By $\mathbf{F}_{\mathrm{q}}^{0}\left(\mathbb{Z}_{+}, \Delta\right)$ we denote a subspace of $\mathbf{B U C}\left(\mathbb{Z}_{+}, \Delta\right)$ which consists of all those sequences $x(j)$ on $\mathbb{Z}_{+}$for which $x(0)=0$ and there exists an $X_{x} \in \mathbf{F}_{q}(\mathbb{Z}, \Delta)$ such that $X_{x}(m)=x(m)$ for all $m \in \mathbb{Z}_{+}$. For such $x$, we define the convolution condition $\mathbf{S}=\mathcal{S} * x$ by

$$
(\mathcal{S} * x)(m)= \begin{cases}\rho(j) x(m-j), & \text { if } m \geqslant j \\ 0, & \text { if } 0 \leqslant m<j .\end{cases}
$$

Lemma 3.1. For each $m \geqslant 0$, the sequence defined by (3.1) acts on $\mathbf{F}_{\mathbf{q}}^{0}\left(\mathbb{Z}_{+}, \Delta\right)$.

Proof. Let $\mathrm{m}>0$ be fixed, $x \in \mathbf{F}_{\mathrm{q}}^{0}\left(\mathbb{Z}_{+}, \Delta\right)$, and $\tilde{x}=\mathcal{S}(\mathrm{m}) x$. Then there exists an $X_{x} \in \mathbf{F}_{q}(\mathbb{Z}, \Delta)$ such that $X_{x}=Y_{x}+Z_{x}$ with $Y_{x} \in P_{q}(\mathbb{Z}, \Delta), Z_{x} \in C_{0}(\mathbb{Z}, \Delta)$, and $x=Y_{x}+Z_{x}$ on $\mathbb{Z}_{+}$. Define $\tilde{Y}_{x}$ and $\tilde{Z}_{x}$ by

$$
\tilde{Y}_{x}(\mathfrak{j})=\left(\rho(m) Y_{x}\right)(\mathfrak{j}), \quad j \in \mathbb{Z}_{+}
$$

and

$$
\tilde{Z}_{x}(\mathfrak{j})= \begin{cases}\left(\rho(m) Z_{x}\right)(j), & \text { if } j \geqslant m, \\ -\left(\rho(m) Y_{x}\right)(j), & \text { if } j<m,\end{cases}
$$

respectively. It is clear that $\tilde{Y}_{x} \in \mathbf{P}_{\mathbf{q}}(\mathbb{Z}, \Delta)$ and $\tilde{Z}_{x} \in \mathbf{C}_{0}(\mathbb{Z}, \Delta)$. Furthermore, if $m=0$, then

$$
\tilde{Y}_{x}(0)+\tilde{Z}_{x}(0)=\left(\rho(0) Y_{x}\right)(0)+\left(\rho(0) Z_{x}\right)(0)=0 .
$$

On the other hand, it is clear that $\tilde{\chi}=\tilde{Y}_{x}+\tilde{Z}_{x}$ on $\mathbb{Z}_{+}$. Hence, $\tilde{x} \in \mathbf{F}_{\mathbf{q}}^{0}\left(\mathbb{Z}_{+}, \Delta\right)$, which completes the proof.

Having in mind the "infinitesimal generator" of the discrete semigroup in continuous case, letting G be the infinitesimal generator of $\mathbf{S}$, then $\mathbf{G}=\mathcal{S}(1)-\mathrm{I}$, where $\mathcal{S}(1)$ is the algebraic generator of $\mathbf{S}$. Therefore, for discrete semigroups, the Taylor formula of order one can be written as

$$
\mathcal{S}(\mathfrak{j}) \mathfrak{u}-\mathfrak{u}=\sum_{k=0}^{j-1} \mathcal{S}(k) G u, \quad \forall j \in \mathbb{Z}_{+}, \quad j \geqslant 1, \quad \forall \mathfrak{u} \in \Delta .
$$

Lemma 3.2. Assume that $\mathrm{G}$ is the infinitesimal generator of $\mathbf{S}$ and let $\mathbf{u}, x \in \mathbf{F}_{\mathbf{q}}^{0}\left(\mathbb{Z}_{+}, \Delta\right)$. Then the following two statements are equivalent.

(1) $\mathrm{u} \in \mathrm{D}(\mathrm{G})$ and $\mathrm{Gu}=-\mathrm{x}$.

(2) $u(j)=\sum_{k=0}^{j} \rho(j-k) \chi(k)$ for all $j \in \mathbb{Z}_{+}$.

Proof. (1) $\Rightarrow$ (2) Using Taylor formula (3.2), we have

$$
\mathcal{S}(\mathfrak{j}) \mathfrak{u}-\mathfrak{u}=\sum_{m=0}^{j-1} \mathcal{S}(\mathrm{m}) \mathrm{Gu}=-\sum_{m=0}^{j-1} \mathcal{S}(\mathrm{m}) x .
$$

Hence, for every $j \in \mathbb{Z}_{+}$,

$$
\mathfrak{u}(\mathbf{j})=(\mathcal{S}(\mathfrak{j}) \mathfrak{u})(\mathfrak{j})+\sum_{m=0}^{j-1}(\mathcal{S}(m) x)(\mathfrak{j})=\rho(j) u(0)+\sum_{m=0}^{j-1} \rho(m) x(j-m)=\sum_{k=0}^{j} \rho(j-k) x(k) .
$$


$(2) \Rightarrow(1)$ Let $j \geqslant 1$. Successively, one has

$$
\begin{aligned}
G u(j) & =(\mathcal{S}(1)-I) \mathfrak{u}(\mathfrak{j})=\rho(1) \mathfrak{u}(\mathfrak{j}-1)-\mathfrak{u}(\mathfrak{j})=\sum_{l=0}^{j-1} \rho(j-l) x(l)-\mathfrak{u}(\mathfrak{j}) \\
& =\sum_{l=0}^{j} \rho(j-l) x(l)-\rho(j-j) x(\mathfrak{j})-\mathfrak{u}(\mathfrak{j})=-x(\mathfrak{j}) .
\end{aligned}
$$

The proof is complete.

Theorem 3.3. Let $\mathbf{T}$ be a q-periodic discrete semigroup of bounded linear operators on the Banach space $\Delta$. If $\mathbf{T}$ is uniformly exponentially stable, i.e., (2.1) is satisfied, then for each $\mathbf{x} \in \mathbf{F}_{\mathbf{q}}^{0}\left(\mathbb{Z}_{+}, \Delta\right)$ the series $\sum_{k=0}^{j} \rho(j-k) x(k) \in$ $\mathbf{F}_{\mathbf{q}}^{0}\left(\mathbb{Z}_{+}, \Delta\right)$.

Proof. Since $\mathbf{T}$ is uniformly exponentially stable, the semigroup $\mathbf{S}=\mathcal{S} * x$ defined by (3.1) is exponentially stable. Thus, the generator $G:=\mathcal{S}(1)-\mathrm{I}$ of $\mathbf{S}$ is an invertible operator. Hence, for each $u \in \mathbf{F}_{\mathrm{q}}^{0}\left(\mathbb{Z}_{+}, \Delta\right)$, there exists an $x \in \mathbf{F}_{\mathbf{q}}^{0}\left(\mathbb{Z}_{+}, \Delta\right)$ such that $(\mathcal{S}(1)-\mathrm{I}) \mathbf{u}=-\chi$. An application of Lemma 3.2 completes the proof.

\section{Exponential stability and action of convolution on spaces $\operatorname{AP}_{0}(\mathbb{Z}, \Delta)$ and $\operatorname{AAP}_{0}^{r}(\mathbb{Z}, \Delta)$}

Again recall that if $\mathbf{F}(\mathbb{Z}, \Delta)$ is a suitable Banach space, then the family of operators $\{\mathcal{S}(j)\}_{\mathfrak{j} \in \mathbb{Z}_{+}}$, denoted by $\mathbf{S}$, defined by

$$
(\mathcal{S} * x)(j)= \begin{cases}\rho(r) x(j-r), & \text { if } j \geqslant r, \\ 0, & \text { if } 0 \leqslant j<r,\end{cases}
$$

is called the evolution semigroup associated with $T$ on the space $F(\mathbb{Z}, \Delta)$.

For the spaces $\mathbf{A P}(\mathbb{Z}, \Delta)$ and $\operatorname{AAP}^{\mathbf{r}}(\mathbb{Z}, \Delta)$, we state the following proposition.

\section{Proposition 4.1.}

(1) If for any $v$ in $\Delta$, the sequences $\rho(j) v$ and $x(\mathbf{j})$ are in $\mathbf{A P}(\mathbb{Z}, \Delta)$ for every $\mathbf{j} \in \mathbb{Z}_{+}$, then $\mathcal{S} * x$ defined by (4.1) also belongs to $\mathbf{A P}(\mathbb{Z}, \Delta)$.

(2) If $x(\mathbf{j}) \in \mathbf{A P}(\mathbb{Z}, \Delta)$ and $\rho(\mathfrak{j}) v \in \mathbf{A A P}^{\mathbf{r}}(\mathbb{Z}, \Delta)$ for every $\mathbf{j} \in \mathbb{Z}_{+}$and all $v \in \Delta$, then $\mathcal{S}(\mathbf{j}) x \in \mathbf{A A P}^{\mathbf{r}}(\mathbb{Z}, \Delta)$.

Proof. (1) Let

$$
p_{m}(j)=\sum_{k=0}^{m} c_{k} e^{i \mu_{k} j} v_{k}
$$

with $c_{k} \in \mathbb{C}, \mu_{k} \in \mathbb{R}, j \in \mathbb{Z}$, and $v_{k} \in \Delta$ such that $p_{m}(s)$ converges uniformly to $x(s)$ for all $s \in \mathbb{Z}$. Then $\rho(\mathfrak{j}) p_{m}(s-\mathfrak{j})$ converges uniformly to $\rho(\mathfrak{j}) x(s-\mathfrak{j})$ for all $s \in \mathbb{Z}$. Since the map

$$
s \rightarrow \rho(j) p_{m}(s-j)=\sum_{k=0}^{m} c_{k} e^{i \mu_{k}(s-j)} \rho(j) \nu_{k}
$$

is almost periodic, its limit $\rho(\mathfrak{j}) \chi(\cdot-\mathfrak{j})$ is also almost periodic.

(2) Let $\left(s_{m}\right)$ be a sequence of real numbers. Given $\rho(j) v \in \mathbf{A A P}^{r}(\mathbb{Z}, \Delta)$ for every $n \in \mathbb{Z}_{+}$and all $v \in \Delta$. Since the sequence $e^{i \mu\left(s_{m}-j\right)}$ is bounded in $\mathbb{C}$, we can suppose that the sequence $\rho(j) e^{i \mu\left(s_{m}-j\right)} v$ converges in $\Delta$ and so the sequence $\rho(j) \sum_{k=0}^{N} c_{k} e^{i \mu_{k}\left(s_{m}-j\right)} v_{k}$ converges in $\Delta$. Let $p_{N}(s-j)=\sum_{k=0}^{N} c_{k} e^{i \mu_{k}(s-j)} v_{k}$ be such that $p_{N}(s-j) \rightarrow x(s-j)$ uniformly for $s \in \mathbb{Z}$. Let $\epsilon>0$ and $N_{0} \in \mathbb{Z}_{+}$be such that the inequality

$$
M_{\omega} e^{\omega j}\left\|x\left(s_{m}-j\right)-p_{N_{0}}\left(s_{m}-j\right)\right\|<\frac{\epsilon}{2}
$$


holds for $j$ sufficiently large. Let us denote by $u_{j}$ the limit of the sequence $\rho(j) p_{N_{0}}\left(s_{m}-j\right)$. Then, for sufficiently large $j$, we have

$$
\begin{aligned}
\left\|\rho(\mathfrak{j}) x\left(s_{m}-\mathfrak{j}\right)-\mathfrak{u}_{\mathfrak{j}}\right\| & \leqslant\left\|\rho(\mathfrak{j}) x\left(s_{m}-\mathfrak{j}\right)-\rho(\mathfrak{j}) p_{N_{0}}\left(s_{m}-\mathfrak{j}\right)\right\|+\left\|\rho(\mathfrak{j}) p_{N_{0}}\left(s_{m}-\mathfrak{j}\right)-u_{j}\right\| \\
& \leqslant M_{\omega} e^{\omega j}\left\|x\left(s_{m}-j\right)-p_{N_{0}}\left(s_{m}-j\right)\right\|+\left\|\rho(\mathfrak{j}) p_{N_{0}}\left(s_{m}-j\right)-u_{j}\right\| \\
& <\epsilon .
\end{aligned}
$$

Hence, the map $\rho(\mathfrak{j}) \chi(\cdot-\mathfrak{j})$ has relatively compact range. This completes the proof.

Denote by $\mathbf{A}_{0}\left(\mathbb{Z}_{+}, \Delta\right)$ the set of all sequences $x(j)$ for which there exist a $j_{x} \in \mathbb{Z}_{+}$and an $X_{x} \in$ $\operatorname{AP}\left(\mathbb{Z}_{+}, \Delta\right)$ such that $X_{x}\left(j_{x}\right)=0$ and

$$
x(\mathfrak{j})= \begin{cases}0, & \text { for all } 0 \leqslant j \leqslant j_{x} \\ x_{x}(j), & \text { if } j>j_{x} .\end{cases}
$$

The smallest closed subspace of $\mathbf{B U C}\left(\mathbb{Z}_{+}, \Delta\right)$ which contains $\mathbf{A}_{0}\left(\mathbb{Z}_{+}, \Delta\right)$ will be denoted by $\mathbf{A P}_{0}\left(\mathbb{Z}_{+}, \Delta\right)$.

Proposition 4.2. If $\chi(\mathfrak{j}) \in \mathbf{A P}_{0}\left(\mathbb{Z}_{+}, \Delta\right)$, then the evolution semigroup $\mathbf{S}$ given in (4.1) acts on $\mathbf{A P}_{0}\left(\mathbb{Z}_{+}, \Delta\right)$.

Proof. The proof is similar to that of [11, Lemma 3.1], and hence is omitted.

The following theorem gives the uniform exponential stability of discrete semigroup $\mathbf{T}$ on $\mathbf{A P}_{0}\left(\mathbb{Z}_{+}, \Delta\right)$.

Theorem 4.3. Let $\mathbf{T}$ be a discrete semigroup on $\Delta$. The following four statements are equivalent.

(1) $\mathbf{T}$ is uniformly exponentially stable.

(2) The convolution condition $\mathbf{S}$ associated to $\mathbf{T}$ on $\mathbf{A P}_{0}\left(\mathbb{Z}_{+}, \Delta\right)$ is uniformly exponentially stable.

(3) The series $\sum_{k=0}^{j} \rho(j-k) x(k) \in \mathbf{A P}_{0}\left(\mathbb{Z}_{+}, \Delta\right)$ for each $x \in \mathbf{A P}_{0}\left(\mathbb{Z}_{+}, \Delta\right)$.

(4) For each $x \in \mathbf{F}_{\mathbf{q}}^{0}\left(\mathbb{Z}_{+}, \Delta\right)=\mathbf{P}_{\mathbf{q}}(\mathbb{Z}, \Delta) \oplus \mathbf{C}_{0}(\mathbb{Z}, \Delta)$, the series $\sum_{k=0}^{j} \rho(j-k) \chi(k) \in \mathbf{F}_{\mathbf{q}}^{0}\left(\mathbb{Z}_{+}, \Delta\right)$.

Proof. (1) $\Rightarrow(2)$ The proof is simple, and so is omitted.

$(2) \Rightarrow$ (3) Assume that $\mathbf{S}$ is uniformly exponentially stable. It means that 1 is not an eigenvalue of $\mathcal{S}(1)$, i.e., $\mathcal{S}(1)-\mathrm{I}$ is invertible. Hence, for each $\mathrm{y} \in \mathbf{A P}_{0}\left(\mathbb{Z}_{+}, \Delta\right)$, there exists an $x \in \mathbf{A P}_{0}\left(\mathbb{Z}_{+}, \Delta\right)$ such that $(\mathcal{S}(1)-\mathrm{I}) \mathrm{y}=-\chi$. On the other hand, it follows from Lemma 3.2 that, for every $j \in \mathbb{Z}_{+}, y(j)=$ $\sum_{k=0}^{j} \rho(j-k) x(k)$. Then, by Proposition $4.2, \sum_{k=0}^{j} \rho(j-k) x(k) \in \mathbf{A P}_{0}\left(\mathbb{Z}_{+}, \Delta\right)$.

$(3) \Rightarrow(4)$ It is obvious.

$(4) \Rightarrow(1)$ This is a direct consequence of Theorem 3.3. The proof is complete.

From Proposition 4.2, we can obtain the following result.

\section{Proposition 4.4. If}

(1) the sequence $\rho(\mathfrak{j}) \in \mathbf{A P}_{0}\left(\mathbb{Z}_{+}, \Delta\right)$ for all $\mathbf{j} \in \mathbb{Z}_{+}$and each $v \in \Delta$;

(2) for each $v \in \Delta, \lim _{j \rightarrow 0} \rho(j) v=v$, uniformly for $j \in \mathbb{Z}_{+}$,

then the semigroup $\mathbf{S}$ given in (4.1) acts on $\mathbf{A A P}_{0}^{\mathbf{r}}\left(\mathbb{Z}_{+}, \Delta\right)$.

Let us denote by $\operatorname{AAP}_{0}^{r}\left(\mathbb{Z}_{+}, \Delta\right)$ the subspace of $\operatorname{AAP}^{r}(\mathbb{Z}, \Delta)$ which consists of all sequences $x(j)$ for which there exists an $X_{x} \in \operatorname{AAP}^{r}(\mathbb{Z}, \Delta)$ such that $X_{x}(0)=0$ and $X_{x}=x$ on $\mathbb{Z}_{+}$. On the basis of Theorem 4.3, we can state the following theorem which gives the uniform exponential stability of discrete semigroup on $\mathbf{A A P}_{0}^{\mathbf{r}}\left(\mathbb{Z}_{+}, \Delta\right)$.

Theorem 4.5. Let $\mathbf{T}$ be a discrete semigroup on $\Delta$. The following statements are equivalent. 
(1) $\mathbf{T}$ is uniformly exponentially stable.

(2) The convolution condition $\mathbf{S}$ associated to the family $\mathbf{T}$ on $\mathbf{A A P} \mathbf{P}_{0}^{\mathbf{r}}\left(\mathbb{Z}_{+}, \Delta\right)$ is uniformly exponentially stable.

(3) The operator $\mathcal{S}(1)$ - I is an invertible operator.

(4) For every $x \in \mathbf{A A P}_{0}^{\mathrm{r}}\left(\mathbb{Z}_{+}, \Delta\right)$, the series $\sum_{k=0}^{j} \rho(j-k) \chi(k) \in \mathbf{A A P}_{0}^{\mathrm{r}}\left(\mathbb{Z}_{+}, \Delta\right)$.

(5) For each $x \in \mathbf{F}_{\mathbf{q}}^{0}\left(\mathbb{Z}_{+}, \Delta\right)=\mathbf{P}_{\mathbf{q}}(\mathbb{Z}, \Delta) \oplus \mathbf{C}_{0}(\mathbb{Z}, \Delta)$, the series $\sum_{k=0}^{j} \rho(j-k) x(k) \in \mathbf{F}_{\mathbf{q}}^{0}\left(\mathbb{Z}_{+}, \Delta\right)$.

In the following, we give a concrete example.

Example 4.6. Consider the discrete Cauchy problem

$$
\left\{\begin{array}{l}
\vartheta_{j+1}=\rho(1) \vartheta_{j}+f(j+1), \quad j \in \mathbb{Z}_{+} \\
\vartheta_{0}=0
\end{array}\right.
$$

The solution of this Cauchy problem is

$$
\vartheta_{j}=\sum_{k=0}^{j} \rho(j-k) f(k),
$$

where $\{\rho(\mathfrak{j})\}_{\mathbf{j} \in \mathbb{Z}_{+}}$is a q-periodic discrete semigroup of bounded linear operators. By virtue of Theorem 4.5 , we can state the following corollary as a conclusion.

Corollary 4.7. The system $u_{j+1}=\rho(1) u_{j}$ is uniformly exponentially stable if and only if for every $f \in \mathbf{A A P}_{0}^{r}\left(\mathbb{Z}_{+}, \Delta\right)$ the solution of Cauchy problem $(\rho(1), 0)$ belongs to $\mathbf{A A P}_{0}^{\mathbf{r}}\left(\mathbb{Z}_{+}, \Delta\right)$.

\section{Acknowledgment}

This research is supported by NNSF of P. R. China (Grant Nos. 61503171, 61403061, and 11447005), CPSF (Grant No. 2015M582091), NSF of Shandong Province (Grant Nos. ZR2016JL021 and ZR2012FL06), DSRF of Linyi University (Grant No. LYDX2015BS001), and the AMEP of Linyi University, P. R. China.

\section{References}

[1] N. Ahmad, H. Khalid, A. Zada, Uniform exponential stability of discrete semigroup and space of asymptotically almost periodic sequences, Z. Anal. Anwend., 34 (2015), 477-484. 1

[2] S. Balint, On the Perron-Bellman theorem for systems with constant coefficients, An. Univ. Timişoara Ser. Ştiinţ. Mat., 21 (1983), 3-8. 1

[3] A. S. Besicovitch, Almost periodic functions, Dover Publications, Inc., New York, (1955). 1

[4] C. Buşe, On the Perron-Bellman theorem for evolutionary processes with exponential growth in Banach spaces, New Zealand J. Math., 27 (1998), 183-190. 1

[5] C. Buşe, D. Barbu, Some remarks about the Perron condition for $\mathrm{C}_{0}$-semigroups, An. Univ. Timişoara Ser. Mat.-Inform., 35 (1997), 3-8. 1

[6] C. Buşe, A. Khan, G. Rahmat, A. Tabassum, Uniform exponential stability for discrete non-autonomous systems via discrete evolution semigroups, Bull. Math. Soc. Sci. Math. Roumanie (N.S.), 57 (105) (2014), 193-205. 1

[7] C. Buşe, A. Zada, Dichotomy and boundedness of solutions for some discrete Cauchy problems, Topics in operator theory, Volume 2, Systems and mathematical physics, Oper. Theory Adv. Appl., Birkhäuser Verlag, Basel, 203 (2010), 165174. 1

[8] C. Corduneanu, Almost periodic oscillations and waves, Springer, New York, (2009). 1

[9] Ju. L. Daleckiǐ, M. G. Kreǐn, Stability of solutions of differential equations in Banach space, Translated from the Russian by S. Smith, Translations of Mathematical Monographs, American Mathematical Society, Providence, R.I., (1974). 1

[10] G. Greiner, J. Voigt, M. Wolff, On the spectral bound of the generator of semigroups of positive operators, J. Operator Theory, 5 (1981), 245-256. 1 
[11] A. Khan, G. Rahmat, A. Zada, On uniform exponential stability and exact admissibility of discrete semigroups, Int. J. Differ. Equ., 2013 (2013), 4 pages. 1, 4

[12] M. G. Kreǐn, On some questions related to the ideas of Lyapunov in the theory of stability, (Russian) Uspehi Matem. Nauk (N. S.), 3 (1948), 166-169. 1

[13] Vu Quoc Phong, On stability of $\mathrm{C}_{0}$-semigroups, Proc. Amer. Math. Soc., 129 (2001), 2871-2879. 1

[14] M. Reghiş, C. Buşe, On the Perron-Bellman theorem for $\mathrm{C}_{0}$-semigroups and periodic evolutionary processes in Banach spaces, Ital. J. Pure Appl. Math., 4 (1998), 155-166. 1

[15] J. M. A. M. van Neerven, Individual stability of $\mathrm{C}_{0}$-semigroups with uniformly bounded local resolvent, Semigroup Forum, 53 (1996), 155-161. 1

[16] J. van Neerven, The asymptotic behaviour of semigroups of linear operators, Operator Theory: Advances and Applications, Birkhäuser Verlag, Basel, (1996). 1

[17] Y.-F. Wang, A. Zada, N. Ahmad, D. Lassoued, T.-X. Li, Uniform exponential stability of discrete evolution families on space of p-periodic sequences, Abstr. Appl. Anal., 2014 (2014), 4 pages. 1

[18] A. Zada, A characterization of dichotomy in terms of boundedness of solutions for some Cauchy problems, Electron. J. Differential Equations, 2008 (2008), 5 pages. 1

[19] A. Zada, N. Ahmad, I. U. Khan, F. M. Khan, On the exponential stability of discrete semigroups, Qual. Theory Dyn. Syst., 14 (2015), 149-155. 1 